\title{
THE WATER VAPOR ABUNDANCE IN ORION KL OUTFLOWS ${ }^{1}$
}

\author{
José Cernicharo, ${ }^{2}$ Javier R. Goicoechea, ${ }^{3}$ Fabien Daniel, ${ }^{2}$ Mercedes R. Lerate, ${ }^{4,5}$ Michael J. Barlow, \\ Bruce M. Swinyard, ${ }^{4}$ Ewine F. van Dishoeck, ${ }^{6}$ Tanya L. Lim, ${ }^{4}$ Serena Viti, ${ }^{5}$ and Jeremy Yates ${ }^{5}$ \\ Received 2006 May 17; accepted 2006 August 7; published 2006 September 8
}

\begin{abstract}
We present the detection and modeling of more than 70 far-IR pure rotational lines of water vapor, including the ${ }^{18} \mathrm{O}$ and ${ }^{17} \mathrm{O}$ isotopologues, toward Orion KL. Observations were performed with the Long Wavelength Spectrometer in Fabry-Pérot mode $(\lambda / \Delta \lambda \sim 6800-9700)$ on board the Infrared Space Observatory between $\sim 43$ and $197 \mu \mathrm{m}$. The water line profiles evolve from P Cygni-type profiles (even for the $\mathrm{H}_{2}^{18} \mathrm{O}$ lines) to pure emission at wavelengths above $\sim 100 \mu \mathrm{m}$. We find that most of the water emission/absorption arises from an extended flow of gas expanding at $25 \pm 5 \mathrm{~km} \mathrm{~s}^{-1}$. Nonlocal radiative transfer models show that much of the water excitation and line profile formation is driven by the dust continuum emission. The derived beam-averaged water abundance is $(2-3) \times 10^{-5}$. The inferred gas temperature $T_{k}=80-100 \mathrm{~K}$ suggests that (1) water could have been formed in the "plateau" by gas-phase neutral-neutral reactions with activation barriers if the gas was previously heated (e.g., by shocks) to $\geq 500 \mathrm{~K}$, and/or (2) $\mathrm{H}_{2} \mathrm{O}$ formation in the outflow is dominated by in situ evaporation of grain water-ice mantles, and/or (3) $\mathrm{H}_{2} \mathrm{O}$ was formed in the innermost and warmer regions (e.g., the hot core) and was swept up in $\approx 1000 \mathrm{yr}$, the dynamical timescale of the outflow.
\end{abstract}

Subject headings: infrared: ISM — ISM: individual (Orion) — ISM: lines and bands — ISM: molecules — line: identification — radiative transfer

Online material: color figure

\section{INTRODUCTION}

Star-forming regions are associated with violent phenomena such as cloud collapse, molecular outflows, and related shocked regions. Under these conditions the neutral gas is warm, and water vapor is predicted to be abundant (Draine \& Roberge 1982) and to play a dominant role in the thermal balance (Neufeld \& Kaufman 1993). Unfortunately, ground-based observations of $\mathrm{H}_{2} \mathrm{O}$ are difficult, so the determination of water column densities is not straightforward. Nevertheless, ground-based observations of water maser lines have been performed toward Orion KL. From VLBI observations of the $6_{16}-5_{23}$ line at $\sim 22 \mathrm{GHz}$, Genzel et al. (1981) determined the kinematics and an expansion velocity for the socalled low-velocity outflow $\left(\sim 18 \pm 2 \mathrm{~km} \mathrm{~s}^{-1}\right)$. The widespread nature of water vapor has been probed with maps of the $3_{13}-2_{20}$ line at $\sim 183 \mathrm{GHz}$ (Cernicharo et al. 1990, 1994). This was the first time that the water abundance was estimated in the different large-scale components around Orion KL. In addition, the high excitation conditions of the "plateau" gas (a mixture of outflows, shocks, and interactions with the ambient cloud) have been revealed by observations of the $5_{15}-4_{22}$ line at $\sim 325 \mathrm{GHz}$ (Menten et al. 1990; Cernicharo et al. 1999a). Due to their maser nature, the water abundance determination from these lines is quite involved. Even the observation of the $\mathrm{H}_{2}^{18} \mathrm{O} 3_{13}-2_{20}$ line at $\sim 203 \mathrm{GHz}$ gives only poor estimates due to the overlap with other molecular lines (Jacq et al. 1988). The HDO species has also been detected and modeled toward Orion KL (Jacq et al. 1990; Pardo

\footnotetext{
${ }^{1}$ Based on observations with $I S O$, an ESA project with instruments funded by ESA Member States (especially the PI countries: France, Germany, the Netherlands, and the UK) and with the participation of ISAS and NASA.

${ }^{2}$ DAMIR, Instituto de Estructura de la Materia, Consejo Superior de Investigaciones Científicas, Serrano 121, 28006 Madrid, Spain; cerni@damir.iem.csic.es.

${ }^{3}$ LERMA, UMR 8112, CNRS, Observatoire de Paris and Ecole Normale Supérieure, 24 Rue Lhomond, 75231 Paris 05, France.

${ }^{4}$ Rutherford Appleton Laboratory, Chilton, Didcot, Oxford 0X11 0QX, UK.

${ }^{5}$ University College London, Gower Street, London WC1E 6BT, UK.

${ }^{6}$ Leiden Observatory, PO Box 9513, 2300 RA Leiden, Netherlands.
}

et al. 2001), but the specific gas and dust chemistry has to be taken into account to derive $\chi\left(\mathrm{H}_{2} \mathrm{O}\right)$.

Infrared Space Observatory (ISO; Kessler et al. 1996) spectrometers have provided the opportunity to observe many IR $\mathrm{H}_{2} \mathrm{O}$ lines toward bright sources such as Orion KL. In particular, Harwitt et al. (1998) presented eight ISO Long Wavelength Spectrometer (LWS) Fabry-Pérot (FP; Clegg et al. 1996) water lines between $\sim 71$ and $\sim 125 \mu \mathrm{m}$ involving energy levels between 300 and $800 \mathrm{~K}$. They estimated $\chi\left(\mathrm{H}_{2} \mathrm{O}\right) \simeq 5 \times 10^{-4}$; however, the interpretation of these lines should include radiative pumping from IR dust photons. A larger set of weaker IR lines, including those of $\mathrm{H}_{2}^{18} \mathrm{O}$ and $\mathrm{H}_{2}^{17} \mathrm{O}$, is needed to minimize the opacity effects always associated with $\mathrm{H}_{2}^{16} \mathrm{O}$ lines. Orion KL has also been targeted with the Short Wavelength Spectrometer (SWS), tracing a smaller region than that probed by the LWS beam below $\sim 45 \mu \mathrm{m}$ (van Dishoeck et al. 1998). Nineteen water absorption lines with energies between 200 and $750 \mathrm{~K}$ were detected (Wright et al. 2000). These authors suggested that mid-IR water lines arise from the low-velocity outflow and estimated $\chi\left(\mathrm{H}_{2} \mathrm{O}\right)=(2-5) \times 10^{-4}$ (for an assumed temperature of 200-350 K). Most recently, the $1_{10}-1_{01}$ lines of $\mathrm{H}_{2}^{16} \mathrm{O}$ and $\mathrm{H}_{2}^{18} \mathrm{O}$ have been observed with the Submillimeter Wave Astronomy Satellite $($ SWAS $)\left[\chi\left(\mathrm{H}_{2}^{16} \mathrm{O}\right) \simeq 3.5 \times 10^{-4}\right.$; Melnick et al. 2000; Snell et al. 2000] and $\operatorname{Odin}\left[\chi\left(\mathrm{H}_{2}^{16} \mathrm{O}\right) \simeq\right.$ $10^{-5}$ to $10^{-4}$; Olofsson et al. 2003]. In these observations, the $\mathrm{H}_{2}^{16} \mathrm{O}$ ground-state line shows widespread emission over a $\sim 5^{\prime}$ region. In this Letter we present all water lines detected in the first far-IR line survey toward Orion KL carried out with the ISO LWS (Lerate et al. 2006) and the radiative transfer models that fit the water data set (involving levels up to $2000 \mathrm{~K}$ ).

\section{OBSERVATIONS AND DATA REDUCTION}

All water lines within the range of the ISO LWS $(\sim 43-197 \mu \mathrm{m})$ were observed using long integrations with the FP spectrometer (L04 AOT), which provides the largest spectral resolution for the LWS instrument. Preliminary results were presented by Cernicharo 

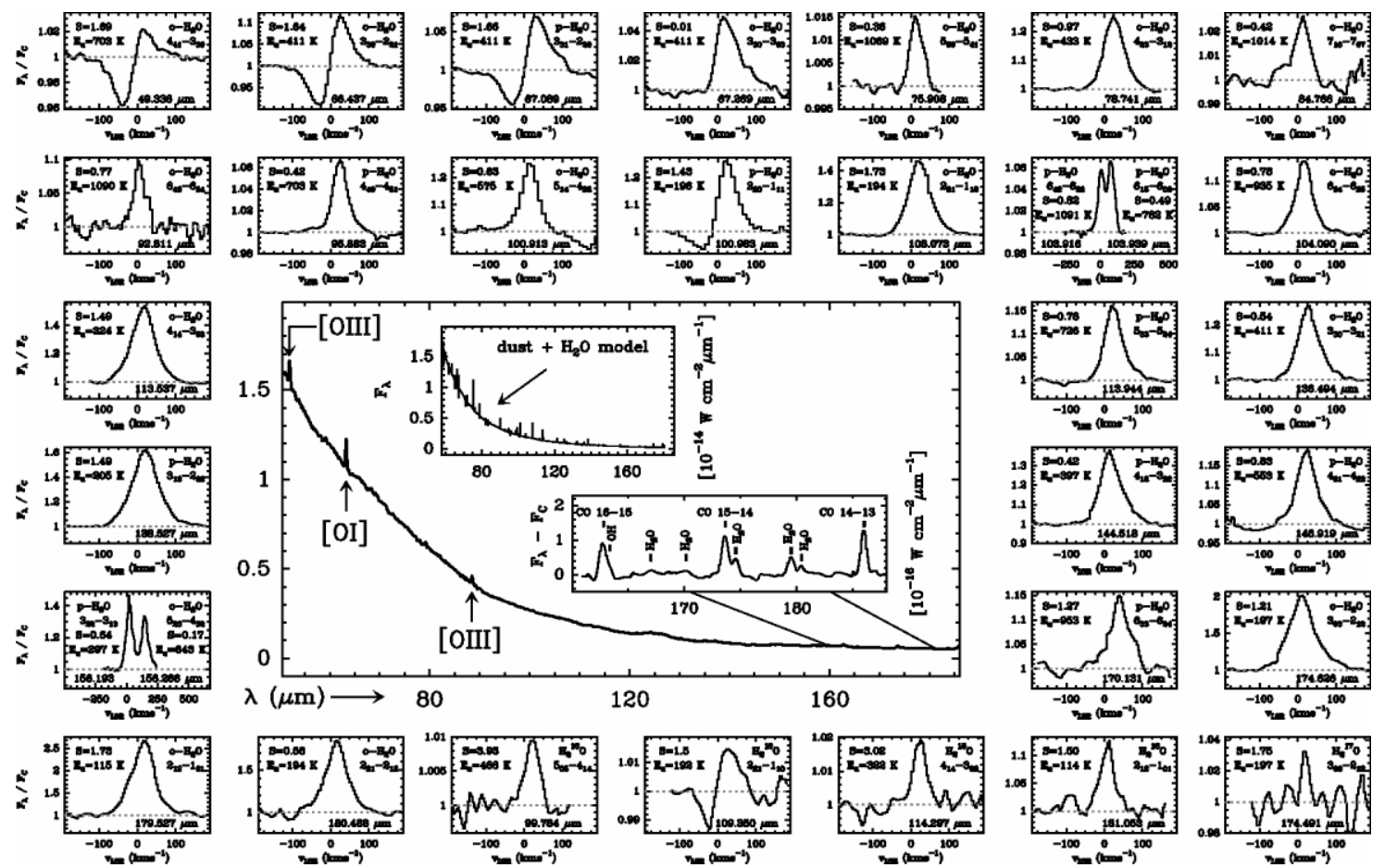

FIG. 1.-Summary of the 70 far-IR water lines detected by the ISO LWS FP interferometer toward Orion KL. The ordinate corresponds to the continuum normalized flux and the abscissa to the velocity (in $\mathrm{km} \mathrm{s}^{-1}$ ). Transition rotational numbers, rest wavelengths (in $\mu \mathrm{m}$ ), upper level energies (in $\mathrm{K}$ ) and intrinsic line strengths are shown in each box. The central inset shows the ISO LWS-grating observations toward Orion at a resolution of $\lambda / \Delta \lambda \sim 300$. The ordinate corresponds to the absolute flux (in $\mathrm{W} \mathrm{cm}^{-2} \mu \mathrm{m}^{-1}$ ) and the abscissa to the wavelength (in $\mu \mathrm{m}$ ). Main molecular features between $\sim 160$ and $\sim 197 \mu \mathrm{m}$ are labeled in a zoom to the grating spectrum. The full radiative transfer model for the far-IR continuum and water line spectrum is also shown in the upper inset.

et al. (1999b). In addition, a complete, less sensitive (L03 AOT), far-IR line survey of Orion has been carried out. Adding both data sets,, more than 70 water lines have been detected (Lerate et al. 2006). The LWS circular aperture size is $\sim 80^{\prime \prime}$, although it slightly depends on the particular LWS detector. In its FP mode the instrumental response is close to a broad-wing Lorentzian with a spectral resolution of $\lambda / \Delta \lambda \sim 6800-9700$. Processing of the water lines from AOT L03 was carried out using the Offline Processing (OLP) pipeline and the LWS Interactive Analysis (LIA) package version 10. AOT L04 spectra were analyzed using the ISO Spectral Analysis Package (ISAP). ${ }^{7}$ The full description of the complex data calibration and reduction process and associated target dedicated time numbers, and all the tabulated line observations and spectroscopic parameters are given by Lerate et al. (2006).

\section{RESULTS AND DISCUSSION}

A summary of the resulting water lines is shown in Figure 1. From these far-IR observations it is clear that water lines show a complicated behavior. For wavelengths above $\sim 100 \mu \mathrm{m}, \mathrm{H}_{2}^{16} \mathrm{O}$ lines are observed in emission. However, for shorter wavelengths, lines arising from energy levels below $\sim 1000 \mathrm{~K}$ and with large line strengths show P Cygni profiles with emission covering a large velocity range. However, those with weak line strengths or those arising from higher energy levels are observed in pure emission. $\mathrm{H}_{2}^{18} \mathrm{O}$ lines also show P Cygni-type profiles below $\sim 100 \mu \mathrm{m}$. In the $\mathrm{H}_{2}^{18} \mathrm{O}$ case, the absorption component is deeper than in the analogous $\mathrm{H}_{2}^{16} \mathrm{O}$ transition. Since optical depth effects are much less severe in $\mathrm{H}_{2}^{18} \mathrm{O}$ lines, the associated P Cygni-type profiles trace the main origin of far-IR water lines toward Orion

${ }^{7}$ ISAP is a joint development by the LWS and SWS Instruments Teams and Data Centers. Constributing institutes are CESR, IAS, IPAC, MPE, RAL, and SRON.
$\mathrm{KL}$, i.e., an extended outflow. Pure emission $\mathrm{H}_{2} \mathrm{O}$ lines peak around $v \simeq 20 \mathrm{~km} \mathrm{~s}^{-1}$ (the source $v_{\mathrm{LSR}}$ is $\sim 9 \mathrm{~km} \mathrm{~s}^{-1}$; Scoville et al. 1983), but it is likely that the most opaque lines are redshifted due to self-absorption. On the other hand, water lines detected below $\sim 50 \mu \mathrm{m}$ are observed in pure absorption with a velocity peak of $v \simeq-10 \mathrm{~km} \mathrm{~s}^{-1}$ (the same applies to most water lines observed by ISO SWS; Wright et al. 2000).

The turnover point between absorption and emission lines is an important clue to interpreting this large data set and determining the relations between continuum + line opacity, line strengths, spatial distribution of gas, and physical conditions. ISO observations clearly show that most of the water vapor detected in the IR arises from a flow of gas expanding at $25 \pm 5 \mathrm{~km} \mathrm{~s}^{-1}$. The inferred expansion velocity is consistent with the low-velocity outflow originally revealed by $\sim 22 \mathrm{GHz} \mathrm{H}_{2} \mathrm{O}$ maser motions (Genzel et al. 1981), but a contribution from the extended high-velocity outflow could be present (Cernicharo et al. 1994). However, no highvelocity line-wing emission is observed at ISO's sensitivity and signal-to-noise ratio. Similar conclusions have been found for the $\mathrm{OH}$ excited rotational lines (Goicoechea et al. 2006).

The main problem with modeling $I S O$ data is the limited spatial resolution, which makes it difficult to constrain the size and origin of the water region. Besides, any detailed fit to the data requires a detailed knowledge of the geometry and of the relative distribution of dust continuum and water lines. Fortunately, high angular resolution maps of water at $\sim 183$ and $\sim 325 \mathrm{GHz}$ lines do reveal the spatial and velocity distribution of water in Orion (Cernicharo et al. 1994, 1999a). In particular, Cernicharo and coworkers detected at least four different water components: the extended "ridge" (extended quiescent gas), the plateau (including the high- and low-velocity outflows), and the very narrow and strong features associated with the small water bullets observed at $\sim 22 \mathrm{GHz}$ (Genzel et al. 1981). 

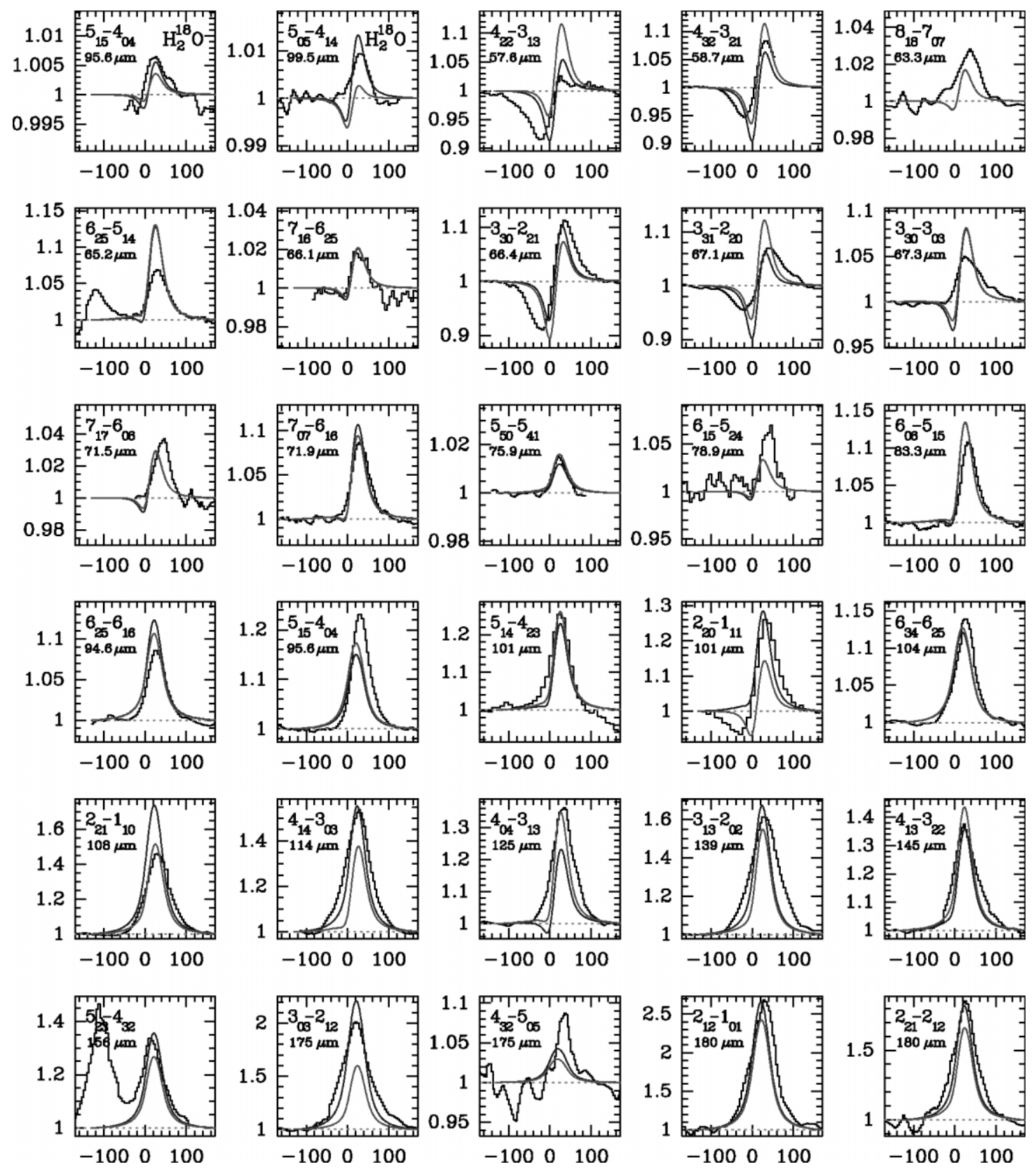

FIG. 2.-Radiative transfer model results discussed in the text. Selected synthetic line profiles (solid curves) are shown over the observed ISO LWS FP detections (histograms). Model parameters are $\chi\left(\mathrm{H}_{2}^{16} \mathrm{O}\right)=3 \times 10^{-5}, n\left(\mathrm{H}_{2}\right)=3.5 \times 10^{5} \mathrm{~cm}^{-3}$ and $T_{k}=80 \mathrm{~K}$ (blue) and $\chi\left(\mathrm{H}_{2}^{16} \mathrm{O}\right)=2 \times 10^{-5}, n\left(\mathrm{H}_{2}\right)=2.5 \times 10^{5} \mathrm{~cm}^{-3}$, and $T_{k}=100 \mathrm{~K}(\mathrm{red})$. The ordinate corresponds to the continuum normalized flux and the abscissa to the velocity (in $\mathrm{km} \mathrm{s}^{-1}$ ). Synthetic line profiles have been convolved with a Gaussian beam of $80^{\prime \prime}$ and with a spectral resolution characterized by a Lorentzian with a width of $33 \mathrm{~km} \mathrm{~s}^{-1}$. [See the electronic edition of the Journal for a color version of this figure.]

In addition, the newly detected far-IR water lines associated with the highest energy levels may have a contribution from the "hot core" (dense and hot inner regions). However, the large far-IR line-plus-continuum opacity will probably hide most of the hot core emission. Finally, radiative transfer effects in the most opaque lines, for example, self-absorption and/or scattering by a lower density diffuse halo may possibly occur at velocity scales not resolved by $I S O$.

In order to estimate the water abundance and the physical conditions prevailing in the expanding gas, we have modeled the first 30 rotational levels of both ortho- and para- $\mathrm{H}_{2} \mathrm{O}$ using the same nonlocal code for lines and dust continuum as in the analysis of $\mathrm{H}_{2} \mathrm{O}$ toward Sgr B2 (Cernicharo et al. 2006). The code has been described elsewhere (González-Alfonso \& Cer- nicharo 1993) and has been recently improved to take into account a more sophisticated description of the dust emissivity and radiative transfer (F. Daniel et al. 2006, in preparation). The dust continuum emission has a crucial role in the radiative excitation of light species such as $\mathrm{H}_{2} \mathrm{O}$ or $\mathrm{OH}$ with transitions in the far-IR and has to be correctly taken into account. In our model, level populations are consistently computed in statistical equilibrium considering collisional excitation and pumping by line and continuum photons. Collisional rates were scaled from those of $\mathrm{H}_{2} \mathrm{O}-\mathrm{He}$ collisions (Green et al. 1993). A three-component model resembling the hot core, the plateau, and the ridge is adopted. In this work we assume that most of the farIR water lines arise only from the plateau. Of course, a minor contribution in the lowest excitation water lines may come from 
the more extended regions (producing narrow line emission), which we do not model here. A low water abundance of (1-8) $\times 10^{-8}$ has been estimated in these regions from SWAS and Odin observations (Snell et al. 2000; Olofsson et al. 2003), and thus the expected contribution to far-IR $\mathrm{H}_{2} \mathrm{O}$ lines is small. In order to have the closest view of the IR radiation field seen by water vapor, we have simultaneously tried to reproduce the full SWS/LWS/ISO continuum emission between $\sim 10$ and $\sim 197 \mu \mathrm{m}$. We find that the continuum level for $\lambda>80 \mu \mathrm{m}$ is well reproduced by considering an inner $10^{\prime \prime}$ IR continuum source (the hot core) simulated by a graybody, with a color temperature of 200-250 K, which is optically thick in the far$\operatorname{IR}\left(\tau_{\lambda}=10 \times 150 / \lambda\right)$. The dust continuum emission from this hot core is attenuated by the surrounding components that we now describe. Taking into account the spatial extent of the 183 and $325 \mathrm{GHz}$ water emission observed at higher spatial resolution (Cernicharo et al. 1990, 1994, 1999a), we model the plateau as a $\sim 40^{\prime \prime}$ diameter unresolved shell expanding at $25 \pm 5 \mathrm{~km} \mathrm{~s}^{-1}$, where water molecules and dust grains coexist ( $T_{d}=T_{k}$ is assumed). The radiative effects caused by an additional, only dust, $5^{\prime}$ diameter component (the ridge), with $T_{d} \simeq 25 \mathrm{~K}$ and $n\left(\mathrm{H}_{2}\right) \simeq 10^{5} \mathrm{~cm}^{-3}$, have also been included to fully match the SWS/LWS continuum level. Dust absorption coefficients are computed from tabulated optical grain properties from Draine \& Lee (1984), in both the plateau and the ridge. Due to the large optical depth of $\mathrm{H}_{2}^{16} \mathrm{O}$ lines in the plateau, we have first modeled the $\mathrm{H}_{2}^{18} \mathrm{O}$ lines to obtain a more accurate estimate of the water abundance with less opacity constraint and faster level population convergence. $\mathrm{A}{ }^{16} \mathrm{O} /{ }^{18} \mathrm{O}$ abundance ratio of 500 has been adopted. After a reasonable fit of the $\mathrm{H}_{2}^{18} \mathrm{O}$ lines, we iteratively search for the best physical conditions that enable to simultaneously reproduce the $\mathrm{H}_{2}^{16} \mathrm{O}$ and $\mathrm{H}_{2}^{18} \mathrm{O}$ spectra. Figure 2 shows two representative models that qualitatively and quantitatively reproduce the majority of observed line profiles and intensities. The best models are found for a plateau temperature, density, and water vapor abundance of $T_{k}=80-100 \quad \mathrm{~K}, \quad n\left(\mathrm{H}_{2}\right)=(2.5-3.5) \times 10^{5} \mathrm{~cm}^{-3}, \quad$ and $\chi\left(\mathrm{H}_{2}^{16} \mathrm{O}\right)=(2-3) \times 10^{-5}$, respectively. The physical conditions inferred from water lines in the plateau are similar to those obtained from OH lines (Goicoechea et al. 2006), which suggests that far-IR lines from both species trace the same expanding gas. The derived water vapor abundance, $\gtrsim 2 \times$ $10^{-5}$, is obviously an averaged value over the large LWS ISO beam and probably indicates that water can be locally more abundant, for example, in the warmer interaction surfaces where the expanding gas shocks the dense ambient material. Larger angular resolution is needed to resolve the relative continuum and $\mathrm{H}_{2} \mathrm{O}$ spatial distribution over this complex region. Nevertheless, the inferred gas temperatures, $\sim 100 \mathrm{~K}$, are significantly below the gas temperature $(\sim 300 \mathrm{~K})$ required to activate the gas-phase neutral-neutral reactions converting most of the available oxygen into water (abundances larger than $\sim 10^{-4}$ are then predicted). Taking into account the short dynamical timescale of the outflow, $\sim 1000 \mathrm{yr}$, water vapor could have been formed by these neutral-neutral reactions if the gas in the plateau was previously (or is locally) heated to $\geq 500 \mathrm{~K}$, for example, by a C-shock passage (Bergin et al. 1998). However, if the plateau gas temperature has reached a maximum temperature of only $\sim 100 \mathrm{~K}$, other formation mechanisms are required to explain the observed water vapor abundances. In particular, the temperature inferred from ISO observations is similar to the water-ice evaporation temperature; therefore, in situ evaporation of water-ice grain mantles formed in an evolutionary stage prior to the onset of the outflow(s) could now dominate the water vapor formation in the plateau. Finally, the observed $\mathrm{H}_{2} \mathrm{O}$ could have also been formed in the innermost and warmest regions (e.g., the hot core) and been swept up by the outflow.

To conclude, despite the large number of detected far-IR excited water lines, neither high gas temperature nor highdensity conditions are required to populate the higher energy water rotational levels. Radiative pumping due to the strong IR radiation field from the cloud is enough to populate these levels, at least in the average picture given by ISO observations. Future observations with Herschel will allow one to map many far-IR water resolved lines with improved angular resolution. It will then be possible to study in great detail the role of water in star-forming regions.

We are grateful to the LWS teams for the quality of the provided data. We thank our referee for useful comments and suggestions. We thank the Spanish DGES and PNIE grants AYA2000-1784, ESP2001-4516, and AYA2003-2785. J. R. G. is supported by a Marie Curie Intra-European Individual Fellowship within the European Community FP6, contract MEIFCT-2005-515340.

\section{REFERENCES}

Bergin, E. A., Melnick, G. J., \& Neufeld, D. A. 1998, ApJ, 499, 777

Cernicharo, J., Goicoechea, J. R., Pardo, J. R., \& Asensio-Ramos, A. 2006, ApJ, 642, 940

Cernicharo, J., Gonzalez-Alfonso, E., Alcolea, J., Bachiller, R., \& John, D. 1994, ApJ, 432, L59

Cernicharo, J., Pardo, J. R., González-Alfonso, E., Serabyn, E., Phillips, T. G., Benford, D. J., \& Mehringer, D. 1999a, ApJ, 520, L131

Cernicharo, J., Thum, C., Hein, H., John, D., Garcia, P., \& Mattioco, F. 1990, ApJ, 231, L15

Cernicharo, J., et al. $1999 \mathrm{~b}$, The Universe as Seen by ISO ed. P. Cox \& M. F. Kessler (ESA-SP 427; Noordwijk: ESA), 565

Clegg, P. E., et al. 1996, A\&A, 315, L38

Draine, B. T., \& Lee, H. M. 1984, ApJ, 285, 89

Draine, B. T., \& Roberge, W. G. 1982, ApJ, 259, L91

Genzel, R., et al. 1981, ApJ, 244, 884

Goicoechea, J. R., et al. 2006, ApJ, 641, L49

González-Alfonso, E., \& Cernicharo, J. 1993, A\&A, 279, 506

Green, S., Maluendes, S., \& McLean, A. D. 1993, ApJS, 85, 181

Harwit, M., et al. 1998, ApJ, 497, L105
Jacq, T., Henkel, C., Walmsley, C. M., Jewell, P. R., \& Baudry, A. 1988, A\&A, 199, L5

Jacq, T., Walmsley, C. M., Henkel, C., Baudry, A., Mauersberger, R., \& Jewell, P. R. 1990, A\&A, 228, 447

Kessler, M. F., et al. 1996, A\&A, 315, L27

Lerate, M. R., et al. 2006, MNRAS, 370, 597

Melnick, G. J., et al. 2000, ApJ, 539, L87

Menten, K. M., et al. 1990, ApJ, 363, L27

Neufeld, D. A., \& Kaufman, M. J. 1993, ApJ, 418, 263

Olofsson, A. O. H., et al. 2003, A\&A, 402, L47

Pardo, J. R., Cernicharo, J., Herpin, F., Kawamura, J., Kooi, J., \& Phillips, T. G. 2001, ApJ, 562, 799

Scoville, N., Kleinmann, S. G., Hall, D. N. B., \& Ridgway, S. T. 1983, ApJ, 275,201

Snell, R. L., et al. 2000, ApJ, 539, L93

van Dishoeck, E. F., et al. 1998, ApJ, 502, L173

Wright, C. M., van Dishoeck, E. F., Black, J. H., Feuchtgruber, H., Cernicharo, J., González-Alfonso, E., \& de Graauw, Th. 2000, A\&A, 358, 689 